\title{
Comunicación

\section{Características estructurales de la producción ALAIC. Una aproximación al conocimiento comunicativo del GT-17}

\author{
GuSTAVO A. LEÓN DUARTE*
}

El presente estudio rescata algunas conclusiones de trabajos previos del autor, que tienen por objeto conocer e identificar las características estructurales que definen a la producción de conocimiento en comunicación, en el interior de la producción científica de los Grupos de Trabajo (GT-17) y de la Asociación Latinoamericana de Investigadores de la Comunicación (ALAIC) entre 1998 y 2004.

Dentro de la relación y el peso que objetiva la estructura de las prácticas y estrategias científicas en la producción del GT 17 de la ALAIC, se destaca la relación e influencia existente entre su capital científico y la principal perspectiva de estudio de la comunicación con la que se identifica el colectivo estudiado: la perspectiva sociocultural y transdisciplinaria en el estudio de la comunicación en Latinoamérica.

PALABRAS CLAVE: investigación de la comunicación, ética, epistemología, transdisciplina.
This paper follows the lead of some of the findings of the author's previous research, which seeks to learn about and identify the structural characteristics that define the production of knowledge on communication within the research done by the Working Teams GT (Grupos de Trabajo) of the Latin American Association of Communication Researchers (ALAIC) between 1998 and 2004. Among the salient features of the relationship and the weight that embody the structure of the scientific practices and strategies in the production of GT-17 of ALAIC is the relationship between its scientific assets and the main perspective of communication studies embraced by the collective under study: a socio-cultural and cross-disciplinary perspective of the study of communication in Latin America.

KEY WORDS: research, comunication ethics, epistemology, cross-discipline.

* Universidad de Sonora, México.

Correo electrónico: gustavol@guaymas.uson.mx 


\section{INTRODUCCIÓN}

Después de 1992, nadie duda que la ALAIC ${ }^{1}$ se haya establecido como la principal asociación científica representativa de los y las investigadores(as) de la comunicación en América Latina. Tampoco que, desde 1998, su principal frente de actuación y producción de conocimiento sean los grupos científicos especializados, mejor conocidos como Grupos de Trabajo (GT). Estos surgen originalmente de forma incipiente en el II Congreso de la ALAIC de 1994, que se realizó en el Departamento de Estudios de Comunicación Social de la Universidad de Guadalajara, en México, bajo la coordinación de los profesores Raúl Fuentes Navarro y Enrique Sánchez Ruiz. No es hasta el IV Congreso de la ALAIC de 1998, realizado en la Universidad Católica de Pernambuco, en Recife, Brasil, cuando los GT entran en la dinámica formal y permanente que hasta el día de hoy presentan. Las sesiones de los GT han permitido desde entonces un debate plural, que converge en un intercambio de experiencias entre los(as) autores(as) y en una mayor integración entre los y las investigadores(as) latinoamericanos.

Para los órganos de dirección de la ALAIC, el proceso de creación, formación y consolidación de los GT ha significado la mejor estrategia encontrada para lograr su misión como entidad científica en el área de la comunicación. Los GT han logrado reunir, de manera sistematizada, los principales trabajos resultantes de las investigaciones realizadas por los estudiosos latinoamericanos de las ciencias de la comunicación en las más diversas universidades del continente, constituyéndose así en un espacio convergente para el debate y el intercambio de experiencias

La ALAIC fue creada en noviembre de 1978 por un grupo de investigadoras e investigadores comprometidos con el avance del campo de la comunicación en América Latina: Patricia Anzola, Elizabeth Safar, Eleazar Díaz Rangel, Antonio Pasquali, Fernando Reyes Matta, Luis Ramiro Beltrán, Jesús Martín-Barbero, Marco Ordóñez, Mario Kaplún, Oswaldo Capriles, Rafael Roncagliolo, Luis Aníbal Gómez, Luiz Gonzaga, Alejandro Alfonso, Joseph Rota y José Marques de Melo, entre otros. Surge bajo una naturaleza gremial y opta por una composición mixta, agrupando asociaciones, instituciones e investigadores(as). 
entre los autores de las investigaciones desarrolladas en este campo del conocimiento. Durante los siete años de vida formal que tienen los GT (1998-2004), se han agrupado, como ponentes en torno a ellos, 389 investigadoras e investigadores, en promedio. Su mayor participación se ha logrado en el Congreso Bianual de 2004, donde participaron 523 ponentes, contemplándose en este año toda una diversidad temática y de perspectivas, así como una multiplicidad de debates en el interior de cada GT (cuadro 1).

CUADRO 1

PARTICIPACIÓN DE PONENTES EN LOS GT DE LA ALAIC

\begin{tabular}{cccccc} 
GT-ALAIC & \multicolumn{3}{c}{ Participación por congresos bianuales } & Participación \\
\cline { 2 - 4 } & 1988 & 2000 & 2002 & 2004 & promedio \\
Totales & 254 & 374 & 405 & 523 & 389
\end{tabular}

Fuentes: Elaboración propia sobre la base de datos de coordinadores de los GT y Boletín -electrónico- ALAIC. Años 2001, 2002, 2003, 2004.

Los GT se constituyen, pues, en espacios para debatir los grandes temas de la contemporaneidad presentes en los estudios de comunicación. Por lo general, dichas discusiones y debates son resultados de investigaciones realizadas en las diversas universidades y en centros de investigación. (M. Krohling, 2005: 3) Entre 1998 y 2004 parece quedar claro que con la iniciativa de creación de los GT se define una apertura novedosa y productiva de gestión para concentrar y sistematizar diferentes experiencias y productos de investigación especializada, que durante décadas habían estado dispersos en el campo académico de la comunicación y fundamentalmente reducidos a algunas personalidades sobresalientes en el ámbito de la producción. Consecuentemente, con la creación de los GT se posibilita la definición de cauces puntuales para la reflexión y la elaboración de las más diversas corrientes y tendencias epistemológicas y metodológicas del pensamiento latinoamericano de la comunicación, a la vez que viabilizan la participación de un mayor número de especialistas, donde cada vez es más notoria la participación 
de jóvenes investigadores(as). En resumen, las propuestas de trabajo específico que implementan los GT de la ALAIC ofrecen sin duda alguna la vía eventual más objetiva y realista para identificar y distinguir de una manera particular y propia el planteamiento de problemas y objetos de investigación, así como los desarrollos epistemológicos y las estrategias metodológicas distintivas del plano de la investigación científica latinoamericana en comunicación.

El presente documento rescata algunas conclusiones de un estudio más amplio, que tiene por objeto conocer e identificar las características estructurales que definen a la producción de conocimiento en comunicación, en el interior de algunas fuentes trascendentales que actualmente configuran el pensamiento latinoamericano de la comunicación, como es el caso, precisamente, de la producción científica del GT-17 de la ALAIC. Por lo tanto, lo aquí expresado se refiere, fundamentalmente, a la producción científica que identifica al GT-17, cuyo nombre formal es Teorías y Metodologías de la Investigación en Comunicación.

\section{EL GT-17: TEORÍAS Y METODOLOGÍAS \\ DE LA INVESTIGACIÓN EN COMUNICACIÓN}

El interés por la producción científica del GT-17 se debe a que es el espacio especializado que concentra, en la ALAIC, las contribuciones referentes al ámbito científico de la producción de conocimiento de la comunicación en América Latina. O mejor aún, porque concentra los trabajos especializados que investigadoras e investigadores de la comunicación latinoamericana realizan exclusivamente en el área de teorías y metodologías de la investigación. De similar manera lo dice la actual coordinadora del GT-17, la profesora investigadora de la Universidad de São Paulo, Brasil, Maria Immacolata Vassallo de Lopes, cuando argumenta que la preocupación y los intereses centrales de investigación del GT-17 se concentran en la reflexión de las cuestiones relativas a la producción de conocimiento en el campo de estudios de la comunicación. (Vassallo, 2001: 43). La base de los argumentos discursivos sobre los que, en una buena parte, "descansan" los distintos sentidos de las afirmaciones que en el presente estudio se han obtenido, son 60 producciones científicas presentadas entre 1998 y 2004 en el GT-17 (ver anexo 1). Ello representa, aproximadamente, el 
análisis de siete de cada diez ponencias presentadas en el GT-17 durante el periodo de estudio (cuadro 2).

CUADRO 2

PONENCIAS PRESENTADAS DENTRO DEL GT-17 DE LA ALAIC

\begin{tabular}{lccccc}
\multirow{2}{*}{ Ponencias } & \multicolumn{4}{c}{ Congresos ALAIC 1998-2004 } & Totales \\
\cline { 2 - 4 } & 1998 & 2000 & 2002 & 2004 & \\
Presentadas & 8 & 35 & 22 & 21 & 86 \\
Recopiladas & 2 & 19 & 22 & 21 & 64 \\
Analizadas & 2 & 19 & 19 & 20 & 60 \\
Citas en texto & 2 & 19 & 19 & 19 & 59 \\
Promedio de & & & & & \\
$\quad$ análisis & $25 \%$ & $54.3 \%$ & $86.4 \%$ & $90.5 \%$ & $69.7 \%$
\end{tabular}

Fuente: elaboración propia con base en datos de ALAIC: GT-17: Teorías y Metodología de Investigación de la Comunicación. En: http://alaic.incubadora.fapesp.br/portal/GTs/GT17 (Consultada en mayo de 2005).

En el tratamiento metodológico de la información se empezó por entender que la obra bajo análisis supone centrar la voluntad de saber sobre el establecimiento de relaciones teóricas y prácticas específicas, mismas que permiten una aproximación a la comprensión de las características estructurales que definen la producción de su conocimiento como una práctica científica, tratada aquí como un proceso histórico de conocimiento y re-conocimiento de sus agentes e instituciones. En este sentido, el amarre de la mirada analítica se enfocó sobre cinco categorías de análisis o constructos teóricos-conceptuales que interesa observar, sistematizar y analizar; a saber: 1) quién es el autor, a quién(es) se dirige, quiénes son los protagonistas del texto, qué se dice de ellos, a qué realidad y contexto se dirige; 2) cómo se entiende y desde dónde se estudia la comunicación; 3 ) cuál(es) es la propuesta teórica y metodológica que aporta al estudio de la comunicación y cuál la característica del conocimiento empírico que de ella emerge; 4) cuál(es) es la afirmación ética-política de la propuesta; y, 
5) cuáles son los procedimientos de exclusión, de crítica y opacidad del texto. El proceso de vincular conceptos abstractos con indicadores empíricos se realizó mediante un plan organizado para analizar y sistematizar la información específica que interesó conocer dentro del conjunto de obras bajo estudio. Para dar cuenta de manera clara y puntual de las anteriores categorías, se construyó una metodología particular, relativamente sencilla y práctica de operar, la cual incluyó un instrumento de registro, identificación y clasificación de las categorías de análisis. La metodología, a la que hemos de identificar aquí como Metodología para Identificar las Características Estructurales del Conocimiento Comunicativo (MICECC), tuvo como objetivo observar, identificar y sistematizar las características estructurales que identifican a la producción del GT-17 de la ALAIC².

\section{APROXIMACIÓN A LAS CARACTERÍSTICAS ESTRUCTURALES DEL GT-17 DE LA ALAIC}

Conviene señalar de entrada que las producciones científicas que expone el GT-17 de la ALAIC, en sus cuatro congresos desarrollados entre 1998 y 2004, han resultado ser originales vetas para aproximarse a conocer e identificar las características estructurales que comporta el campo académico de la comunicación en Latinoamérica. Del análisis interpretativo efectuado a las 60 producciones científicas presentadas en este periodo, se ha comprobado que los aportes de las producciones analizadas transitan desde varios países latinoamericanos hasta los más diversos temas, objetos y preocupaciones de estudio: el origen de la institucionalización del campo; la reconstitución teórica y metodológica del campo académico; los fundamentos de su interdisciplinariedad; el estatuto disciplinario de los estudios de la comunicación y la actualización epistemológica; el sentido de la comunicación; los principales aportes metodológicos y las estrategias de implementación; relatos de experiencias metodológicas aplicadas; principales problemáticas que

2 Por motivos de espacio editorial, aquí se obvia la descripción y explicación profunda y propia de la MICECC. Para mayor información, en este sentido, consúltese León (2005, 2005a, 2006a). 
se tiene en los estudios de la comunicación; perspectivas, balances y corrientes de los estudios de la comunicación en Latinoamérica en la era digital, etc.

Pero una aproximación más cuidadosa a las 60 producciones científicas analizadas, nos permite precisar la afirmación anterior: 1) Que la producción científica del GT-17 de la ALAIC proviene de ocho países latinoamericanos.

2) Que la mayor participación procede fundamentalmente de tres países, los cuales concentran más de $78 \%$ de la creación: Brasil con 40\%; México con 20\%, y Argentina con $18.3 \%$. (cuadro 3).

\section{CUADRO 3}

ORIGEN Y PORCENTAJES DE PRODUCCIONES. GT-17 ALAIC

$\begin{array}{cccc}\text { Brasil } & \text { México } & \text { Argentina } & \text { Venezuela } \\ 24=40 \% & 12=20 \% & 11=18.3 \% & 4=6 \% \\ \text { Chile } & \text { Bolivia } & \text { Colombia } & \text { Perú } \\ 3=5 \% & 2=3 \% & 1=1 \% & 1=1 \%\end{array}$

Fuente: elaboración propia con base en datos del GT-17 de la ALAIC. 1998-2004. En: http://alaic.incubadora.fapesp.br/portal/GTs/GT17 (consultada en julio de 2005).

3) Que las líneas de investigación del GT-17 de la ALAIC, esencialmente se concentran en cuatro dominios de intereses: a) la actualización disciplinar (teórica-metodológica) de la comunicación, con $26.6 \%$ de los casos analizados; b) el sentido y la construcción de objetos de investigación en comunicación, con $23.3 \%$; c) perspectivas, balances y retos de la comunicación, con $20.3 \%$; y d) relatos de experiencias metodológicas aplicadas a casos de estudio, con $15.2 \%$ de los casos analizados. (cuadro 4)

La participación específica en la presentación de las 60 producciones científicas analizadas en el seno del GT-17 de la ALAIC también ha perfilado en parte cuál es, en qué consiste y qué tipo de efectos genera la relación y el peso de la distribución del capital científico que objetiva la 


\section{CUADRO 4}

LÍNEAS DE PRODUCCIÓN, NÚMERO Y PARTICIPACIÓN DE PONENCIAS POR PAÍSES EN EL GT-17 DE LA ALAIC 1998-2004.

Líneas de investigación Producciones Año presentación

Actualización disciplinar (teórica-metodológica)

$$
16=26.6 \% \quad 1998,2000,2002,2004 \text {. }
$$

de la comunicación.

Sentido y construcción de objetos de investigación en

$$
14=23.3 \% \quad 2000,2002,2004
$$
comunicación.

Perspectivas, balances y retos de $12=20.3 \%$ $2000,2002,2004$ la comunicación.

Relatos de experiencias me todológicas aplicadas a casos de estudio.

$$
9=15.2 \%
$$

Reconstitución y fundamentación teórica y metodológica de la comunicación.

Institucionalización y crítica al estado de conocimiento.

Otros.

Totales: número y porcentaje:

$$
\begin{array}{cc}
4=6.6 \% & 1998,2000,2004 \\
3=5 \% & 1998,2002 \\
2=3 \% & 2002,2004 \\
60=100 \% & 60=100 \%
\end{array}
$$

Fuente: elaboración propia con base en datos del GT-17 de la ALAIC. 1998-2004. En: http://alaic.incubadora.fapesp.br/portal/GTs/GT17 (consultada en julio de 2005).

estructura del campo de estudios en comunicación en América Latina. Es decir, en cómo se distribuye el reconocimiento o crédito que le es otorgado a un(a) investigador(a) por el conjunto de pares-competidores dentro del propio campo científico; asimismo, en cómo se ve reflejado dicho reconocimiento en la producción discursiva analizada. Por un lado, es de resaltar que, en términos generales, la participación de los y las protagonistas en la producción científica en comunicación que hace suya la ALAIC, no ha tenido una presencia constante en el periodo anali- 
zado. Es decir que existen pocos(as) investigadores(as) que han participado de manera permanente en el interior del GT-17 durante los cuatro congresos ALAIC analizados. Existe, en cambio, un reducido grupo de especialistas que, por los motivos que sean, han tenido una colaboración en al menos tres cuartas partes de los cuatro congresos analizados (es el caso de la profesora venezolana Migdalia Pineda de Alcázar) o, incluso, en $100 \%$ de participación en dichos eventos: la profesora brasileña Immacolata Vassallo de Lopes y el investigador mexicano Raúl Fuentes Navarro.

Tomando también en consideración que tanto Vassallo como Fuentes han participado de manera casi permanente desde la reconstitución de la ALAIC, en 1992, no parece extraño que ahora ambos emerjan entre los principales investigadores(as) latinoamericanos(as) que más reconocimiento explícito han recibido en las contribuciones científicas del GT-17 de la ALAIC; incluso, significativamente por encima de autores latinoamericanos pioneros en el campo y que, tradicionalmente, habían venido recibiendo los mayores índices de reconocimiento hasta el cierre de los años 90.

Sin embargo, lo que parece ser todavía más sorprendente y meritorio, es que tres intelectuales latinoamericanos (Immacolata Vassallo de Lopes, Raúl Fuentes Navarro y Jesús Martín-Barbero) puedan situar su reconocimiento de una manera equivalente o, incluso, por encima de autores occidentales y norteamericanos. Este es un indicador que contradice la tesis manejada por algunos destacados investigadores latinoamericanos: que el pensamiento latinoamericano de la comunicación no ha logrado ser un paradigma dominante en su propia cuna. (Marques de Melo, 1998: 10). Se puede afirmar que la obra de Vassallo de Lopes es la principal fuente de conocimiento y reconocimiento del capital científico en el interior del GT-17 de la ALAIC, al ser su trabajo el más citado dentro del total de las producciones científicas analizadas. (cuadro 5)

Un apoyo metodológico esencial que requirió la perspectiva cualitativa con la que se nutre la MICECC fue el tratamiento cuantitativo de las producciones científicas analizadas mediante un registro bibliométrico, particularmente el registro y uso de citas. El estudio bibliométrico permitió no sólo confirmar y profundizar el conocimiento sobre el volumen de 
las publicaciones, la productividad de los y las autores(as), colectivos y fuentes de publicación, sino, también, del conocimiento y reconocimiento académico y científico que le es otorgado a un(a) investigador(a) por el

\section{CUADRO 5}

RECONOCIMIENTO DE CITAS EN TEXTO POR AUTOR.

PRODUCCIÓN DEL GT-17 DE LA ALAIC. 1998-2004

$\begin{array}{lcc}\text { Autores latinoamericanos } & \text { Número } & \text { Porcentaje } \\ \text { Immacolata Vassallo L. } & 20 & 21.05 \\ \text { Jesús Martín-Barbero } & 17 & 17.90 \\ \text { Raúl Fuentes Navarro } & 16 & 16.84 \\ \text { Guillermo Orozco G. } & 11 & 11.54 \\ \text { Néstor García Canclini } & 8 & 8.42 \\ \text { Antonio Pasquali } & 7 & 7.36 \\ \text { Otros(as) } & 16 & 16.84 \\ \text { Totales } & 95 & 100\end{array}$

Autores occidentales

$\begin{array}{lcc}\text { Edgar Morin } & 16 & 19.04 \\ \text { Pierre Bourdieu } & 14 & 16.66 \\ \text { Armand Mattelart } & 13 & 15.47 \\ \text { Anthony Giddens } & 11 & 13.09 \\ \text { Immanuel Wallerstein } & 10 & 11.90 \\ \text { John B. Thompson } & 8 & 9.52 \\ \text { Otros(as) } & 12 & 14.28 \\ \text { Totales } & 84 & 100\end{array}$

Fuente: elaboración propia con base en datos de la producción científica del GT-17 de la ALAIC. 1998-2004. En: http://alaic.incubadora.fapesp.br/portal/GTs/GT17. (consultada en julio de 2005).

Nota: para determinar el número y orden final de citas se tomaron en cuenta sólo a autores que fueron citados más de cuatro ocasiones dentro del universo de las ponencias analizadas. El número máximo de citas por autor/ponencia fue de dos. En total se obtuvieron 179 referencias: 95 de ellas correspondieron a autores(as) latinoamericanos y 84 a autores(as) occidentales. 
conjunto de pares-competidores. Ello fue imprescindible para entender la relación y el peso que objetiva la estructura de las prácticas científicas en la producción del GT-17 de la ALAIC, y así distinguir la relación e influencia existente entre principales autores latinos citados y la principal perspectiva de estudio de la comunicación con la que se identifica el colectivo estudiado: la perspectiva sociocultural de la comunicación. Esta pluralidad de personajes en torno a una perspectiva de estudio de la comunicación va pasando gradualmente a primer plano a medida que se ajusta tanto la visión histórica del conjunto de estrategias del grupo cuanto mayor avance temporal se tiene en la presentación de las ponencias. Luego, habría además que reconocer el fuerte sostén teórico de empatía que suponen los autores occidentales dentro del conjunto de estrategias discursivas que apuntalan la perspectiva sociocultural y transdisciplinaria en el estudio de la comunicación en Latinoamérica.

De manera general, se podría decir que las estrategias discursivas que decididamente impulsan Vassallo y Fuentes (entre muchos autores y autoras más), apuntan a significar y a fundamentar que en la actualidad la investigación latinoamericana en comunicación inserta sus objetos de estudio en el espacio de las ciencias sociales y de las humanidades, utilizando para ello, principalmente, el enfoque sociocultural de la comunicación. Las implicaciones de este enfoque de estudio son múltiples, y exigen abrir el campo de estudios de la comunicación a otras concepciones epistemológicas y éticas para dar solución a los diversos problemas complejos que plantean las distintas sociedades latinoamericanas. Lo que no significa que los trabajos presentados en el GT-17 de la ALAIC tengan un patrón homogéneo de estudio, de temáticas o de reconocimiento de ciertos personajes $\mathrm{y}$, por tanto, de exención de autores o exclusión de perspectivas de análisis. Por el contrario, lo que se trata de significar es que si la homogeneidad no viene suministrada por unas obvias unidades de tiempo, de espacio, de temáticas o de personajes, entonces tal vez hubiera que buscarla en el conjunto de referencias y estrategias discursivas internas que los diversos agentes han desplegado en la producción ALAIC analizada, bajo las circunstancias históricas y contextuales específicas del colectivo. Es este un primer sentido de nuestra voluntad de saber.

Conviene desde ya aclarar una primera conclusión general que es válida para aprender a comprender las características estructurales de la 
producción científica en comunicación que se produce en el GT-17 de la ALAIC: que su investigación definitivamente representa un sentido de cambio y distinción respecto de la producción científica que caracteriza, por ejemplo, a la llamada Escuela Latinoamericana de la Comunicación (ELACOM) y, en menor medida, a la producción que sobre este tema publican de una década a esta parte las principales revistas científicas de comunicación en Latinoamérica. Pero, ¿en qué consiste este cambio? ¿Cómo opera este cambio y en qué sentido se representa diferente a la producción de la ELACOM O las revistas científicas de comunicación?

Sostenemos aquí, a manera de una segunda conclusión general, que la producción científica de la ALAIC ha sentado bases para adquirir una institucionalización cognitiva particular, al menos en lo que respecta y refleja la producción científica del GT-17. Si bien habría que reconocer que las premisas de partida en su investigación casi siempre apuntan a establecer que el pensamiento latinoamericano de la comunicación está, y debe seguir estando, fuertemente apegado a un pensamiento crítico y progresista en la región, como evidentemente se presenta también en la producción de la ELACOM y en las revistas, sus preocupaciones de estudio están fuertemente marcadas por la necesidad de actualizar permanentemente el campo de estudios, sus objetos y métodos de investigación, así como por el desarrollo prospectivo del campo y, esencialmente, el de su investigación.

Efectivamente, se ha identificado que los pensamientos crítico y autorreflexivo de la ALAIC apuntan hacia varios frentes. Respecto a los procesos de desarrollo institucional que se despliegan en el plano cognitivo en el campo, se advierte que en la producción ALAIC existe el sentido de una necesidad casi generalizada entre sus investigadores(as) de que los estudios de la comunicación en América Latina se abran a las ciencias sociales e interactúen de manera más vigorosa y permisible con otros campos de conocimiento. También hacia modelos disciplinares cerrados y herméticos sobre sí mismos, como es, según se apunta sistemáticamente en la producción de la ALAIC analizada, el caso de la comunicación. Pero, también, queda claro que entre los y las protagonistas de la producción ALAIC, existe un consenso discursivo manifiesto que impulsa un enfoque común y compartido (al menos en lo fundamental) de objetivos, metas y métodos específicos para el estudio 
de la comunicación. Tal vez este proceso de institucionalización del conocimiento, contrariamente a lo que se afirma en algunas contribuciones sobresalientes del GT-17 de la ALAIC, aún este verde y lejos de la autopretendida consolidación.

Una tercera conclusión general se da a través de un acuerdo general aplastante y prendido entre los académicos y académicas que hacen suya la producción ALAIC examinada: el sentido evidente de malestar, incertidumbre y desfase que ha presentado el campo académico ante los procesos de institucionalización social en el contexto particular que presentan las distintas sociedades latinoamericanas. En nuestra opinión, la coyuntura pendiente o imperfecta que abren los dos planos de la institucionalización (social y cognitiva) en los estudios de la comunicación en América Latina, parece ser la fuente misma del malestar en el campo. Coincidentemente, en su esencia parece traer consigo, según se desprende del análisis transversal e interpretativo de la producción ALAIC analizada, el efecto más dañino que se le puede atribuir a los estudios de la comunicación para tratar de mejorar un contexto social tan desigual e injusto como el latinoamericano. Justamente es este el devenir del sentido y la preocupación principal de tratar de sustentar la hipótesis de que la investigación de la comunicación en América Latina muestra claras tendencias de una actividad científica vinculada al estudio sobre la realidad sociocultural, como un movimiento emergente de la práctica de las ciencias sociales del siglo XXI, al margen de las adscripciones disciplinarias, burocráticas o pedagógicas, como plantea Raúl Fuentes Navarro y algunos(as) investigadores(as) más de la región.

En particular, una característica estructural que hace notaria y distintiva a la producción de la ALAIC es la premisa de que las cuestiones centrales que patentiza el campo académico de la comunicación, y los desafíos que en la actualidad se le presentan a su investigación, tienen que ver fundamentalmente con su estatuto disciplinario. Parece quedar claro que el estatuto disciplinario de la comunicación, en América Latina, se caracteriza como un campo de conocimiento multidisciplinar $\mathrm{y}$ transdisciplinar que es producto de relaciones convergentes entre su objeto de estudio, la especificidad de sus contribuciones analíticas y la particularidad de la evolución histórica de ambos. El significado de la comunicación desde un enfoque transdisciplinar presenta, entre otras 
implicaciones de estudio, el partir de una "reconstrucción de realidades" al momento de tratar de definir cómo se concibe y desde dónde se aborda el objeto de conocimiento que la comunicación implica. Una primera realidad reclama a la comunicación como el principal detonador de las transformaciones económicas, políticas y culturales de la sociedad actual.

En específico, ha quedado evidenciado que el movimiento de su estatuto transdisciplinar apunta, como lo sostienen principalmente Fuentes y Vassallo, hacia la superación de los límites entre especialidades cerradas y jerárquicas en los campos de conocimientos y el establecimiento de un campo de discurso y prácticas sociales, cuya legitimidad académica y social dependerá cada vez más de la profundidad, extensión, pertinencia y solidez de sus explicaciones o de aquellas propias que se derivan de las exigencias del conocimiento, y no del supuesto prestigio institucional acumulado.

Un punto incuestionable de acuerdo en la producción científica de la ALAIC es el enfoque y el tratamiento de la comunicación como objeto de estudio: la comunicación como un campo de conocimiento con un objeto de estudio transhistórico, eminentemente sociocultural, complejo, multidimensional y centrado en los sujetos y procesos de producción de sentido. Si habría que definir la especificidad del estudio de la comunicación en tanto objeto de estudio, parece quedar claro que esta no sería otra que la plataforma ética-política que sustenta a la comunicación. De acuerdo con la producción científica de la ALAIC analizada, la afirmación disciplinaria de la comunicación en Latinoamérica sólo puede ser sostenida por este consenso y sostenida sobre una propuesta ética-política, pues es justamente aquí donde residen las condiciones de construcción de una especificidad en su objeto. También, se puede concluir que el origen del campo de la comunicación reside en movimientos de convergencia y sobreposición de contenidos y metodologías que no han sido construidos de forma autónoma, sino que se han venido desarrollando de una forma creciente e histórica desde el interior de las ciencias sociales y humanas en las últimas décadas. En el caso específico de Latinoamérica, la posición que identifica a Vassallo se afirma desde el pensamiento transdisciplinar, el cual constituye una perspectiva reciente que se manifiesta en el crecimiento de análisis 
autorreflexivos. La producción del GT-17 de la ALAIC parece confirmar dicha afirmación. Por ejemplo, en el caso de la transdisciplinariedad en la región, su origen no parece ser otro que el proceso histórico de la globalización, según lo han planteado, transversal y cronológicamente, los estudios desarrollados por Vassallo, Fuentes, Pineda, Torrico, Vizer, Grillo, Papalini, López, Barboza, Maldonado, Fasano, Jiménez, Ruiu, Ramírez, Álvarez, Luján, Sanahuja y Cervantes.

Bajo esta perspectiva epistemológica se evidencian algunos otros componentes teóricos y metodológicos, así como aquellos que son propios del sistema de valores ético-políticos que hace suya la producción ALAIC analizada. Uno de ellos es el papel atribuido al sujeto o actor social: el de un ente cognitivamente autónomo; como un practicante autorreflexivo de la comunicación con otros(as) y como interventor moralmente responsable, creador de la misma realidad social en la que vive. Lo que parece, incluso, un buen perfil descriptivo del futuro profesional de la comunicación.

Otro componente básico es el fundamento teórico-metodológico de la comunicación. Su constitución está fuertemente influenciada por el papel que desarrolla el sujeto en tanto que sólo con dichas atribuciones se puede suministrar una forma discursiva a las cuestiones del conocimiento mutuo que los actores emplean de una manera no discursiva en su conducta: de este conocimiento mutuo entre los sujetos depende que las actividades sociales tengan sentido en la práctica. El fundamento epistémico de la comunicación consiste, entonces, en esa producción en común de sentido. Su investigación y teorización no pueden, por tanto, limitarse al estudio de los medios que los sujetos sociales usan para generar el sentido de su actividad y, necesariamente por ello, de su propia identidad. Contextualmente, el sentido que incorpora dicho fundamento epistémico, la reclamada reestructuración del campo de la comunicación y, concretamente, la globalización, implican un cambio de entendimiento en la comunicación que se genera en la ALAIC y que irradia a partir de un polo de dominio a casi todo lo demás: los fundamentos teórico-metodológicos de la perspectiva sociocultural de la comunicación.

Respecto a las características propias que presenta la investigación de la comunicación en Latinoamérica, se identifican algunas tendencias 
que permiten confirmar la hipótesis de que el sentido de la habituación cognitiva del colectivo del GT-17 de la ALAIC se inclina hacia una actividad científica de producción de conocimiento sobre la realidad sociocultural, tal y como lo sugieren Fuentes, Vassallo, Martín-Barbero y otros, y otras protagonistas más. Se parte de estudiar la comunicación como una producción social de sentido sobre la producción social de sentido. El sentido de la comunicación aparece, entonces, como un campo posible de configuración de nociones, visiones, percepciones, y de intenciones, operaciones, acciones; donde se aprende a poner en común lo que se configura como un colectivo sociocultural y se puede y exige intervenir legítimamente en las formas de esa construcción y a configurar otras. En sus prácticas de producción, se parte por entender que la investigación de la comunicación no es ni supone una disciplina o una ciencia propiamente. En la producción ALAIC analizada, las contribuciones generalmente se autoidentifican como un campo de estudio abierto que puede favorecer el enriquecimiento teórico y metodológico de la comunicación social y humana. Específicamente, las características estructurales de la metodología que identifica a la producción ALAIC exteriorizan que el polo de su reflexión ha sido progresivamente desplazado de los medios hacia los grupos sociales que están integrados en prácticas sociales y culturales más amplias. Los y las investigadores(as) de la perspectiva sociocultural del estudio de la comunicación son llevados a tomar en consideración la existencia de verdaderas estrategias de organización discursiva para lograr un recorrido significativo. Un ejemplo de la descripción detallada de este recorrido en la región es el análisis de la recepción, lo que le ha valido el carácter de una etnografía de las audiencias. Es de observar que en los recientes estudios de recepción en América Latina, cuya enfoque trata de lograr una visión integradora y comprensiva que se remonta en la región a la década de los años 70, se reconoce la consolidación de la temática ligada a las culturas populares, donde se tiene como apoyos centrales a la teoría compleja y multifacética de la recepción, y como ejes básicos de reflexión el desplazamiento de los medios a las mediaciones y los procesos de hibridización cultural.

Algunas otras características metodológicas de corte estructural que comporta la producción del GT-17 de la ALAIC, se identifican con la sínte- 
sis creativa y el sentido interpretativo del investigador(a) ante el objeto de estudio, con la contrastación metodológica de los análisis sociohistóricos y discursivos, y con la interpretación y reinterpretación del sentido común. De ahí que sus autorreflexiones evolucionen pausadamente por un análisis que examina, separa, reconstruya y busque descubrir nuevos patrones y recursos en la constitución de la comunicación en Latinoamérica.

También, la perspectiva transdisciplinar de la comunicación con la que se autoidentifica la producción de la ALAIC analizada, presenta como características importantes el considerar la diversidad y la complejidad de dimensiones; la abundancia de contextos y la multiplicidad de aspectos que los procesos y fenómenos comunicacionales tienen; los procedimientos de focalización y delimitación son de carácter dialécticos, flexibles, amplios, heurísticos, heterodoxos, analíticos y hermenéuticos; procura mezclar diversas interpretaciones, modelos y procesos de construcción de conocimiento concreto. La perspectiva transmetodológica se configura, pues, en una dimensión en la cual confluyen ciertos principios de interpretación que exigen ser mediados por una gama de metodologías explicativas, el análisis sociohistórico y la reflexión permanente de los procesos sociohistóricos, culturales y comunicacionales. La confluencia de sus escenarios valora particularmente, por un lado, la producción de sentido y la unidad, pluralidad y multidimensionalidad de contextos; por otro, la apropiación de lógicas y modelos teóricos diversos y alternativos que configuran la confluencia de la mediatización y las estrategias de mediación. (cuadro 6)

No obstante, también habría que indicar que el pasaje de lo transdisciplinar, como mero enunciado formal o retórico, hacia una concepción de investigación crítica y transformadora, implican, tal y como se sostiene anteriormente, un esfuerzo singular de fundamentación teórica y experimentación metodológica que, de acuerdo con la producción ALAIC analizada, y contrariamente a lo que se afirma en ella, aún parece estar en proceso de desarrollo, o, en todo caso, alejada de su pretendida y autoanunciada consolidación.

Por otro lado, un primer acercamiento analítico a la producción científica de la ALAIC deja entrever que nos encontramos, efectivamente, ante una extensa categoría de problemas y retos que están recurrentemente identificados: la actualización disciplinar de la comunicación; el 


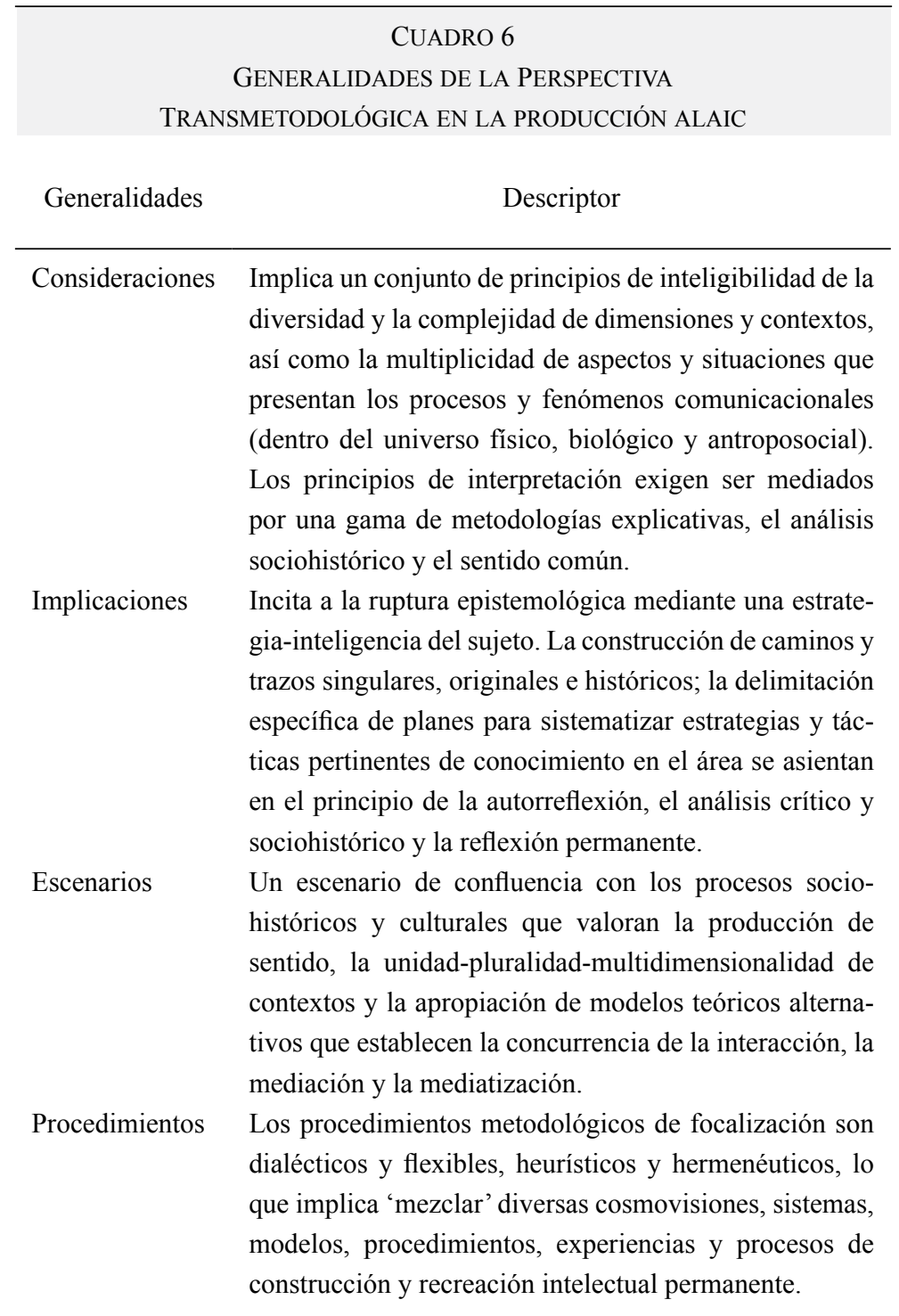

Fuente: elaboración propia con base en datos de: Fuentes (1998; 2000); Maldonado (2000; 2002); Vassallo (1998, 2000, 2004). 
sentido y la construcción de objetos de investigación en comunicación, y el desarrollo de perspectivas, balances y retos de la comunicación. En varias contribuciones de este colectivo se deja entrever que algunos viejos problemas han sido resueltos en forma parcial o casi total, pero, también, que algunos más aún no han sido resueltos del todo ni en forma inicial. Se puede comprobar que además existen en el campo latinoamericano de la comunicación una serie de problemas emergentes que aún no tienen ni un nombre claro, que en muchos casos no se ha iniciado su exploración, o que son, de plano, un enigma por falta de información en el interior de la comunidad académica; es decir, se expresa lo que Jesús Galindo ha llamado carencia de una cibercultura general y generalizada de la información, la comunicación y el conocimiento; en síntesis, hace falta una sociedad de comunicación de alto desarrollo.

Un reto central que enfrenta la investigación latinoamericana en comunicación emerge de la necesidad de incentivar la creatividad e innovación no sólo teórica sino también metodológica, especialmente si se toma en cuenta que las metodologías no son recetas acabadas y que el investigador las puede renovar, mejorar o refutar al tener que adaptarlas a sus temas de investigación. Pues, como ha significado la profesora Pineda de Alcázar, un enfoque metodológico creativo e innovador es realmente aquel que, a partir de una opción metodológica aceptada, es capaz de adaptarla a su investigación para rebasar sus límites y poner en evidencia sus ventajas para abordar unos determinados problemas, pero también sus limitaciones.

Para no perder de vista los retos y las nuevas interrogantes que se plantean en el campo académico de la comunicación en la región, la producción ALAIC ha identificado algunos problemas relevantes en el estudio de la comunicación actual: la globalización y el papel de los medios como instancias socializadoras; la sociedad de la información y los cambios que suponen las tecnologías de la información y la comunicación en los procesos de comunicación y en los mismos medios; la necesidad de desarrollar una teoría de los medios y las mediaciones que integre el lenguaje, las tecnologías, los medios y la sociedad; el desconocimiento de los aportes de las neurociencias y ciencias cognitivas en el desarrollo de la inteligencia artificial y los nuevos impactos y modalidades que se crean en el campo de la comunicación. Justamente 
esto es lo que parece consolidar la formación de nuevas síntesis de disciplinas o de convergencias disciplinares, es decir, de transdisciplinas, que son producto, según ha quedado sistemáticamente reconocido en la producción ALAIC analizada, de las relaciones convergentes entre el objeto de estudio, la especificidad de las contribuciones analíticas y la particularidad de la evolución histórica entre ambos.

Generalmente, no parece extraño encontrar, como colofón en la producción científica de la ALAIC, la propuesta de que el campo académico de la comunicación debe aprovechar positivamente las coyunturas contextuales, epistemológicas y metodológicas que actualmente se abren en las ciencias sociales. Ello supone avanzar decididamente en las exigencias que marca el rigor teórico-metodológico junto a los contextos y tiempos socioculturales de América Latina. Para Vassallo, por ejemplo, actualmente la práctica transdisciplinar de la comunicación puede producirse a través de movimientos de convergencias y de apropiaciones mutuas que incorporan preguntas y saberes históricos, antropológicos y estéticos, al mismo tiempo que la sociología, la antropología y la ciencia política comienzan a interesarse por los medios y los modos como operan las industrias culturales. Así, parece quedar claro que, para conocer adecuadamente la multidimensionalidad de los procesos comunicativos en la sociedad, es necesario aumentar y generalizar, en el campo de la comunicación, el movimiento de autorreflexión para que se extienda hacia todas las ciencias sociales, con particular atención a la reflexión epistemológica crítica, transformadora y actualizada, como marcas distintivas del pensamiento latinoamericano en comunicación.

\section{Bibliografia}

KROHLING, Margarita (2005) "Balance de las actividades directivas 1998-2005”. Boletín Especial ALAIC 2005. En: http://www.eca.usp. br/associa/alaic/boletin22.htm. Consultada en mayo de 2005.

LEÓN, G. (2005) Institucionalización del campo académico de la comunicación en América Latina. Un acercamiento a las características estructurales de la Escuela Latinoamericana de la Comunicación. Trabajo de investigación doctoral. Barcelona: Universidad Autónoma de Barcelona. 1501002895/TR344. 
- (2005a) "Ejercicios de lectura crítica no androcéntrica aplicados al texto académico de la comunicación". Revista Internacional de Comunicación. España: Universidad de Sevilla.

- (2006) "Sobre la institucionalización de la comunicación en América Latina". En R. Fuentes (coord.) La constitución cientifica del campo académico de la comunicación en México y en Brasil: análisis comparativo. Guadalajara: CONACYT.

- (2006a) "Sobre la investigación del campo académico de la comunicación en América Latina. Una aproximación a las características estructurales de la investigación latinoamericana en comunicación". Tesis de doctorado. Barcelona: Universidad Autónoma de Barcelona MARQUeS DE MELO, José (1998) "Prefacio". En José Marques de Melo y Juçara Gorski. A trajetória comunicacional de Luiz Ramiro Beltrán. São Paulo: UNESCO-UMESP. I CELACOM.

VASSALLO, Immacolata (2001) "El campo de la comunicación: reflexiones sobre su estatuto disciplinar. En Comunicación: campo y objeto de estudio. Perspectivas reflexivas latinoamericanas. Guadalajara: ITESO/UC/UAA/UdeG.

\section{Referencias a ponencias que se incluyen} en el análisis del GT-17 de la ALAIC

AGUIRRE Alvis, José Luis (2000) "Enfoques cuantitativos y cualitativos en la investigación comunicacional". Ponencia presentada en el V Congreso de la ALAIC de 2000. Santiago de Chile. En: http:// www.eca.usp.br/alaic/gt17.htm (13.06.2005).

ÁLVAREZ, Adriana (2002) "Cibergrafía: propuesta teórico-metodológica para el estudio de los medios de comunicación social cibernéticos". Ponencia presentada en el VI Congreso de la ALAIC de 2002. Santa Cruz de la Sierra, Bolivia. http://www.eca.usp.br/alaic/materi al\%20congresso\%202002/gtvassallolopes.htm (14.06.2005).

BARBOSA, Marialva (2000) "Comunicação: consolidação de uma interdisciplina como paradigma de construção do campo comunicaciona". Ponencia presentada en el V Congreso de la ALAIC de 2000. Santiago de Chile. En: http://www.eca.usp.br/alaic/gt17.htm (13.06.2005).

- (2002) "Conceitos, armadilhas, olhares: apontamentos metodológicos para a consolidação de um campo transdisciplinar.” Ponencia 
presentada en el VI Congreso de la ALAIC de 2002. Santa Cruz de la Sierra, Bolivia. http://www.eca.usp.br/alaic/material\%20congresso \%202002/gtvassallolopes.htm (14.06.2005).

CANElas Rubim, Antonio Albino (2000) "A contemporaneidade como idade mídia". Ponencia presentada en el v Congreso de la ALAIC de 2000. Santiago de Chile. En: http://www.eca.usp.br/alaic/gt17.htm (13.06.2005).

- (2004) "Espetáculo, cultura e idade mídia". Ponencia presentada en el VII Congreso de la ALAIC de 2004. Buenos Aires, Argentina. http:// alaic.incubadora.fapesp.br/portal/Gts/gt172004 (15.06.2005).

CAPPARELLI, Sérgio y Ida Regina Stumph (2000) "O campo da comunicação revisitado". Ponencia presentada en el V Congreso de la ALAIC de 2000. Santiago de Chile. En: http://www.eca.usp.br/alaic/gt17.htm (13.06.2005).

CARDINALE Baptista, Maria Luiza (2000) "Emoção e subjetividade na paixão-pesquisa em comunicação: desafios e perspectivas metodológicas". Ponencia presentada en el V Congreso de la ALAIC de 2000. Santiago de Chile. En: http://www.eca.usp.br/alaic/gt17.htm (13.06.2005).

- (2002) "A cartografia de processo de escrita. Uma experiência com a metodologia da sensibilidade." Ponencia presentada en el VI Congreso de la ALAIC de 2002. Santa Cruz de la Sierra, Bolivia. http://www. eca.usp.br/alaic/ 2002/gtvassallolopes.htm (14.06.2005).

- (2004) "Comunicação, amorosidade e autopoiese." Ponencia presentada en el VII Congreso de la ALAIC de 2004. Buenos Aires, Argentina. http://alaic.incubadora.fapesp.br/portal/Gts/gt172004 (15.06.2005).

CASTRO, Juana (2002) "Imaginarios: instrumento de análisis de la mediación". Ponencia presentada en el VI Congreso de la ALAIC de 2002. Santa Cruz de la Sierra, Bolivia. http://www.eca.usp.br/alaic/ 202002/ gtvassallolopes.htm (14.06.2005).

CERVANTES Barba, Cecilia (2002) "El grupo de discusión: de la mercadotecnia al estudio de la cultura y la comunicación”. Ponencia presentada en el VI Congreso de la ALAIC de 2002. Santa Cruz de la Sierra, Bolivia. http://www.eca.usp.br/alaic/ 202002/gtvassallolopes. htm (14.06.2005).

CORONA, Sarah (2000) "El estudio de las formas comunicativas como 
disciplinas del cuerpo. Propuesta metodológica". Ponencia presentada en el V Congreso de la ALAIC de 2000. Santiago de Chile. En: http:// www.eca.usp.br/alaic/gt17.htm (13.06.2005).

DEL VALle Rojas, Carlos (2004) "Meta investigación de la comunicación en Chile: 1970-2003". Ponencia presentada en el VII Congreso de la ALAIC de 2004. Buenos Aires, Argentina. http://alaic.incubadora.fapesp.br/portal/Gts/gt172004 (15.06.2005).

ENTEL, Alicia (2000) "El mundo en un detalle". Una antropología dialéctica para los estudios de comunicación y cultura". Ponencia presentada en el V Congreso de la ALAIC de 2000. Santiago de Chile. En: http://www.eca.usp.br/alaic/gt17.htm (13.06.2005).

ESPINOZA, Pablo (2004) "La enseñanza de la metodología y la formación de comunicadores. Sistematización de una experiencia de enseñanza-aprendizaje de la metodología cualitativa en una facultad de comunicación social". Ponencia presentada en el VII Congreso de la ALAIC de 2004. Buenos Aires, Argentina. http://alaic.incubadora.fapesp.br/portal/Gts/gt172004 (15.06.2005).

FASANO, Patricia; Juan Giménez, Aurora Ruiu y Alejandro Ramírez (2002) "La realidad social como pieza comunicacional". Ponencia presentada en el VI Congreso de la ALAIC de 2002. Santa Cruz de la Sierra, Bolivia. http://www.eca.usp.br/alaic/material\%20congresso \%202002/gtvassallolopes.htm (14.06.2005).

FíGARO, Roseli (2000) "A centralidade da categoria trabalho para se refletir sobre o campo da comunicação". Ponencia presentada en el V Congreso de la ALAIC de 2000. Santiago de Chile. En: http://www. eca.usp.br/alaic/gt17.htm (13.06.2005).

- (2002) "Estudos de recepção: comunicação e trabalho versus ação comunicativa." Ponencia presentada en el VI Congreso de la ALAIC de 2002. Santa Cruz de la Sierra, Bolivia.http://www.eca.usp.br/alaic/ material\%20congresso\%202002/gtvassallolopes.htm (4.06.2005).

FUENTES Navarro, Raúl (1998) "Fundamentos teórico-metodológicos de la perspectiva sociocultural para el estudio de la comunicación". Ponencia presentada en el IV Congreso de la ALAIC de 1998. Pernambuco, Brasil. En: http://www.eca.usp.br/alaic/gt17.htm (13.06.2005).

- (2000) "Exploraciones teórico-metodológicas para la investigación sociocultural de los usos de internet." Ponencia presentada en el V 
Congreso de la ALAIC de 2000. Santiago de Chile. En: http://www. eca.usp.br/alaic/gt17.htm (13.06.2005).

- (2002) "Investigación y postgrados en comunicación en México: los desafíos del siglo XXI." Ponencia presentada en el VI Congreso de la ALAIC de 2002. Santa Cruz de la Sierra, Bolivia. En, http://www.eca.usp.br/alaic/material\%20congresso\%202002.htm (4.06.2005).

- (2004) "La documentación académica y la producción de conocimiento en ciencias de la comunicación." Ponencia presentada en el VII Congreso de la ALAIC de 2004. Buenos Aires, Argentina. http:// alaic.incubadora.fapesp.br/portal/Gts/gt172004 (15.06.2005).

GALINDO Cáceres, Jesús (2004) "Sobre Comunicología y Comunicometodología. Primera guía de apuntes sobre horizontes de lo posible". Ponencia presentada en el VII Congreso de la ALAIC de 2004. Buenos Aires, Argentina. http://alaic.incubadora.fapesp.br/portal/ Gts/gt172004 (15.06.2005).

GIL, Gastón Julián (2000) "La epistemología y estudios de comunicación: en busca de la constitución del campo". Ponencia presentada en el V Congreso de la ALAIC de 2000. Santiago de Chile. En: http:// www.eca.usp.br/alaic/gt17.htm (13.06.2005).

GRILLO, Mabel (2000). "Estilos interactivos, auto ubicación espacial e identidades locales". Ponencia presentada en el V Congreso de la ALAIC de 2000. Santiago de Chile. En: http://www.eca.usp.br/alaic/ gt17.htm (13.06.2005).

GUADARRAMA, Luis (2002) "Familias y medios de comunicación. Metodología para orientar la investigación". Ponencia presentada en el VI Congreso de la ALAIC de 2002. Santa Cruz de la Sierra, Bolivia. http://www.eca.usp.br/alaic/material\%20congresso\%202002/gtvassallolopes.htm (4.06.2005).

GUAZINA, Liziane (2004) "O Conceito de mídia na comunicação e na ciência política: desafios interdisciplinares". Ponencia presentada en el VII Congreso de la ALAIC de 2004. Buenos Aires, Argentina. http://alaic.incubadora.fapesp.br/portal/Gts/gt172004 (15.06.2005).

KILPP, Suzana (2004) "Mundos imaginados em Muvuca". Ponencia presentada en el VII Congreso de la ALAIC de 2004. Buenos Aires, 
Argentina. http://alaic.incubadora.fapesp.br/portal/Gts/gt172004 (15.06.2005).

LENARDUZZI, Víctor (2000) "La prohibición de la imaginación". Recepción y usos de la Escuela de Frankfurt en los estudios sobre la comunicación". Ponencia presentada en el V Congreso de la ALAIC de 2000. Santiago de Chile. En: http://www.eca.usp.br/alaic/gt17. htm (13.06.2005).

LEÓN Duarte, Gustavo (2004) "La Escuela Latinoamericana de la Comunicación. Una propuesta metodológica para su estudio". Ponencia presentada en el VII Congreso de la ALAIC de 2004. Buenos Aires, Argentina. http://alaic.incubadora.fapesp.br/portal/Gts/gt172004 (15.06.2005).

- (2006) Sobre la institucionalización del campo académico de la comunicación en América Latina. Una aproximación a las características estructurales de la investigación latinoamericana en comunicación. Tesis de doctorado. Barcelona: Universidad Autónoma de Barcelona.

LOPES, Luiz Carlos (2002) "Percepção e comunicação: mitos e problemas contemporâneos". Ponencia presentada en el VI Congreso de la ALAIC de 2002. Santa Cruz de la Sierra, Bolivia. http://www.eca. usp.br/alaic/material $\% 20$ congresso $\% 202002 /$ gtvassallolopes.htm (14.06.2005).

- (2004) "Os paradigmas da comunicação." Ponencia presentada en el VII Congreso de la ALAIC de 2004. Buenos Aires, Argentina. http://alaic.incubadora.fapesp.br/portal/Gts/gt172004 (15.06.2005).

LuJÁN, Dora y Sanahuja, Sonia (2000) "Construyendo significados con las metodologías". Ponencia presentada en el VI Congreso de la ALAIC de 2002. Santa Cruz de la Sierra, Bolivia. http://www.eca. usp.br/alaic/material\%20congresso $\% 202002 /$ gtvassallolopes.htm (14.06.2005).

- (2002) "Competencia interactiva. Televisión, tiempo y espacio en la vida cotidiana." Ponencia presentada en el VI Congreso de la ALAIC de 2002. Santa Cruz de la Sierra, Bolivia.http://www.eca. usp.br/alaic/material\%20congresso $\% 202002 /$ gtvassallolopes.htm (4.06.2005).

MALDONADO, Alberto Efendy (2000) "Reflexões sobre a pesquisa teórica em comunicação na América Latina”. Ponencia presentada en 
el V Congreso de la ALAIC de 2000. Santiago de Chile. En: http:// www.eca.usp.br/alaic/gt17.htm (13.06.2005).

- (2002) "Productos mediáticos, estrategias, recepción. La perspectiva transmetodológica." Ponencia presentada en el VI Congreso de la ALAIC de 2002. Santa Cruz de la Sierra, Bolivia. http://www.eca. usp.br/alaic/material $\% 20$ congresso $\% 202002 /$ gtvassallolopes.htm (14.06.2005).

MARTINO, Luis C. (2000). "Elementos para uma epistemología da comunicação". Ponencia presentada en el V Congreso de la ALAIC de 2000. Santiago de Chile. En: http://www.eca.usp.br/alaic/gt17.htm (13.06.2005).

- (2004) "Ceticismo e interdisciplinaridade: paradoxos e impasses da teoria da comunicação" Ponencia presentada en el VII Congreso de la ALAIC de 2004. Buenos Aires, Argentina. http://alaic.incubadora. fapesp.br/portal/Gts/gt172004 (15.06.2005).

MATA, Maria Cristina. (2000). "Interrogaciones sobre el público". Ponencia presentada en el V Congreso de la ALAIC de 2000. Santiago de Chile. En: http://www.eca.usp.br/alaic/gt17.htm (13.06.2005).

PAPALINI, Vanina. (2002). "La comunicación: espacio teórico para la ideología contemporánea y su crítica". Ponencia presentada en el VI Congreso de la ALAIC de 2002. Santa Cruz de la Sierra, Bolivia. http://www.eca.usp.br/alaic/material\%20congresso\%202002/gtvassallolopes.htm (14.06.2005).

PINEDA, Migdalia. (2000). "Los procesos de la comunicación a la luz de los medios interactivos: revisiones conceptuales y de tipologías". Ponencia presentada en el V Congreso de la ALAIC de 2000. Santiago de Chile. En: http://www.eca.usp.br/alaic/gt17.htm (13.06.2005).

- (2002) "Sociedad de la información y ciencias de la comunicación: modos de enseñanza e investigación." Ponencia presentada en el VI Congreso de la ALAIC de 2002. Santa Cruz de la Sierra, Bolivia. http://www.eca.usp.br/alaic/material\%20congresso\%202002/gtvassallolopes.htm (14.06.2005).

- (2004) "La investigación de la comunicación en América Latina: cómo lo hacemos y hacia dónde vamos"? Ponencia presentada en el VII Congreso de la ALAIC de 2004. Buenos Aires, Argentina. http:// alaic.incubadora.fapesp.br/portal/Gts/gt172004 (15.06.2005). 
PITTA, Esteban (2002) "Proceso de construcción de un instrumento para evaluar la calidad de la programación infantil". Ponencia presentada en el VI Congreso de la ALAIC de 2002. Santa Cruz de la Sierra, Bolivia. http://www.eca.usp.br/alaic/202002/gtvassallolopes.htm (14.06.2005).

RIzO García, Marta (2004) “La dimensión de la interacción en la comunicología. Apuntes para una reflexión teórica y algunas propuestas pedagógicas". Ponencia presentada en el VII Congreso de la ALAIC de 2004. Buenos Aires, Argentina. http://alaic.incubadora.fapesp. br/portal/Gts/gt172004 (15.06.2005).

ROCHA Da silva, Alexandre (2004) "Pós-mídia: a comunicação das multidões". Ponencia presentada en el VII Congreso de la ALAICde 2004. Buenos Aires, Argentina. http://alaic.incubadora.fapesp. br/portal/Gts/gt172004 (15.06.2005).

RUDIGER, Francisco (2000) "Nietzsche e as origens da teoria do sujeito comunicacional contemporâneo". Ponencia presentada en el V Congreso de la ALAIC de 2000. Santiago de Chile. En: http://www.eca. usp.br/alaic/gt17.htm (13.06.2005).

TAVARES, Denise (2004) "O jornalismo visto através da pesquisa”. Ponencia presentada en el VII Congreso de la ALAIC de 2004. Buenos Aires, Argentina. http://alaic.incubadora.fapesp.br/portal/Gts/ gt172004 (15.06.2005).

TORRICO, Eric (2002) "Las teorías en germen sobre la "nueva sociedad" y la comunicación: Esbozo para una sistematización crítica". Ponencia presentada en el VI Congreso de la ALAIC de 2002. Santa Cruz de la Sierra, Bolivia. http://www.eca.usp.br/alaic/material\%20 congresso\%202002/gtvassallolopes.htm (14.06.2005).

VALENTINO, Alejandra (2004) "El tratamiento de los datos en la investigación en comunicación: aportes desde los estudios del discurso". Ponencia presentada en el VII Congreso de la ALAIC de 2004. Buenos Aires, Argentina. http://alaic.incubadora.fapesp.br/portal/Gts/ gt172004 (15.06.2005).

VARELA, Sebastián; Diego Aguilar y Santiago Barandiarán (2004) "Propedéutica para estudios comparados. La construcción social del miedo en las ciudades de La Plata, Guadalajara y San Juan”. Ponencia presentada en el VII Congreso de la ALAIC de 2004. Bue- 
nos Aires, Argentina. http://alaic.incubadora.fapesp.br/portal/Gts/ gt172004 (15.06.2005).

VARGAS Carrillo, Soledad (2004) "Estilos de vida, ética y estética en los suplementos dominicales de los diarios $A B C$, La Vanguardia y El País (1974-1999)". Ponencia presentada en el VII Congreso de la ALAIC de 2004. Buenos Aires, Argentina. http://alaic.incubadora. fapesp.br/portal/Gts/gt172004 (15.06.2005).

VASSALLO de Lopes, Maria Immacolata (1998) "A construção de uma metodologia multidisciplinar para o estudo de recepção da telenovela". Ponencia presentada en el IV Congreso de la ALAIC de 1998. Pernambuco, Brasil. En: http://www.eca.usp.br/alaic/ gt17.htm (13.06.2005).

- (2000) "O campo da comunicação: reflexões sobre seu estatuto disciplinar" Ponencia presentada en el V Congreso de la ALAIC de 2000. Santiago de Chile. En: http://www.eca.usp.br/alaic/gt17.htm (13.06.2005).

- (2002). "Narrativas televisivas e interculturalidade." Ponencia presentada en el VI Congreso de la ALAIC de 2002. Santa Cruz de la Sierra, Bolivia. http://www.eca.usp.br/alaic/material\%20congresso \%202002/gtvassallolopes.htm (14.06.2005).

- (2004). "Por um paradigma transdisciplinar para o campo da comunicação." Ponencia presentada en el VII Congreso de la ALAIC de 2004. Buenos Aires, Argentina. http://alaic.incubadora.fapesp. br/portal/Gts/gt172004 (15.06.2005).

VIDALES Gonzáles, Carlos E. (2004) "Hacia una reestructuracíon epistemológica de los modelos de comunicación”. Ponencia presentada en el VII Congreso de la ALAIC de 2004. Buenos Aires, Argentina. http://alaic.incubadora.fapesp.br/portal/Gts/gt172004 (15.06.2005).

VIZER, Eduardo (2002) "La trama invisible de la vida social: comunicación, sentido y realidad". Ponencia presentada en el VI Congreso de la ALAIC de 2002. Santa Cruz de la Sierra, Bolivia.http://www. eca.usp.br/alaic/material\%20congresso\%202002/gtvassallolopes. htm (14.06.2005). 